and with the precepts taught by some of the Grecian philosophers, of whom we shall have occasion to speak.

The priests themselves were subject to very strict rules of life. Cleanliness was strictly enforced. They were obliged to wash themselves twice by day, and twice at night, and to cut their hair every third day. Circumcision was also practised among them; and we are told that Pythagoras, desiring to become acquainted with the rules of the Egyptian priests, was obliged to submit to this rite. Woollen clothing was forbidden; linen and cotton only were allowed to be used. Their food consisted of vegetables, and of the flesh of those animals which could be sacrificed to the gods. The selection of certain animals was no doubt made, in part, on sanitary grounds; but also from superstitious reasons, the origin of which cannot be traced out. Cows were never sacrificed, because they were sacred to Isis; but oxen were offered-and therefore their flesh was eaten. Swine were not sacrificed; and their flesh was eaten by the priests only once in each year, at the full moon. Fishes and other marine animals were prohibited. Among vegetables, farinaceous legumes and onions were forbidden; the leguminous plants, either because they were difficult of digestion, or because of some unknown mystic reason. Whether wine was permitted is a question which is not altogether decided.

The hygienic rules which guided the laity, although less severe than those to which the priests were subject, were very strict. Even to the kings, a fixed quantity of food and drink was prescribed; and, in the temple at 'Thebes, an inscribed pillar contained imprecations against Menes, one of the kings, for having broken through the rules of frugality. All the functions, even that of generation, were, as far as could be, subjected to laws, and time was specially fixed for their performance. 'The children went with naked feet, and ate little but fruits, roots, and the marrow of the papyrus. Every Egyptian was compelled each month to undergo a three days course of emetics, purgatives, and enemata ; for, as Herodotus and Diodorns inform us, they imagined disease to arise from excess, and from the retention of undigested food. The result of attention to a hygienic code is shown in the statement of the ancient writers, that the Egyptians were a healthy and long-lived people.

[To be continued.]

\section{OHriainal Communiations.}

\section{MEDICAL CASES.} By C. M. Durnast, M.D., Physician to the East Suffolk and
Ipswich Hospital.

CASE I. Hysteric Somnolency. A young woman, aged 22, was brought to me by her mother, who stated that her daughter had been in the habit, for eight or nine months, of dropping off to sleep constantly during the day; that these attacks would often occur every five minutes; and that, even when walking, she would sometimes stop suddenly and fall asleep. The only history that I could obtain was, that she had been much excited and fatigued by a close attendance upon an epileptic and hysterical sister, and that this somnolency had followed the subsidence of the disease in her sister. She had not been in my room for more than three minutes, when she fell asleep, and remained breathing somewhat heavily for about two or three minutes, when she awoke with a start, and conversed as usual, but as suddenly, during the conversation, she relapsed into sleep, and which recurred six or seven times during the visit. Her countenance was sallow, and her expression heavy and unexcitable; the pupils acted naturally, and were not dilated; she had no headache; the tongue was coated, its edges were indented by the teeth; the pulse was languid, and not accelerated; the extremities were generally cold; the appetite was good, indeed she was always hungry; the catamenia had been suppressed for five months; the bowels were always constipated. She was ordered to use a tepid sponge bath every morning, to be followed by brisk friction; to take a saline mixture, with the compound tincture of valerian; and to take, at night, pills containing calomel, colocynth, colchicum, and croton oil, alternately with pills composed of blue pill, sulphate of zinc, and galbanum. This treatment was continued for some weeks, during which she steadily and uninterruptedly improved in health, and became less somno- lent, and ultimately, the catamenia reappearing, her friends reported her well.

REMARKS. This case is interesting, as illustrating in a marked degree one of the many phases of the hysterical diathesis. The symptoms were clearly attributable to an overexcited and, subsequently, exhausted nervous system, superadded to the powerful influence of imitation; for, on inquiry, I found that the younger sister, aged 12 , in addition to the seizures which appeared to bave been epileptiform, subsequently suffered from hysteric somnolency, although in a less marked degree than obtained in the elder. Dr. Copland refers to this form of hysteria, and states, "that the irritation, whether mental, cerebral, or uterine, seems to induce congestion of the vessels of the head." In the above case, the mental excitement, with its consequent exhaustion, engendered both the suspended menstrual function, and also the impeded cerebral circulation.

Case Ir. Albuminuria, followed by Atonic Peritonitis and Death. Autopsy. A. B., æt. 17, a labourer at an iron foundry, was admitted as an in-patient of the Ipswich Hospital, March 14th, 1860. On admission it was stated that he had been ill only a fortnight; that he had taken cold, and that he had been swollen "all over" since the attack.

On examination, the nature of the disease was at once ap. parent. His face was pallid, pasty, and swollen; the eyelids were cedematous; the feet and legs very much swollen and pitting; the integuments of the abdomen were anasarcous, with an indistinct sensation of fluctuation in its cavity. The tongue was tolerably clean; the pulse feeble, not accelerated; the bowels were confined; the urine was diminished in quantity, and becoming almost solid on the addition of heat and nitric acid. The heart's action was feeble, but the sounds and rhythm were unaffected, respiration was accelerated, with some œdematous crepitation at the base of the left lung.

$\mathrm{He}$ had ridden to the hospital in an open cart, the day being very cold. He was at once sent to bed; and as the dropsy was of such recent occurrence, he was ordered to lose a few ounces of blood by cupping over the kidneys. In addition to this, citrate of ammonia, with spirit of nitric ether, was prescribed.

March 15th. He was much in the same condition. The difficulty of breathing was increased, which appeared to be rather the result of increasing debility than of additional effusion. The bowels had acted several times very copiously without aperient medicine; the stools were bilious and watery. Pulse feeble, but not much accelerated. He was ordered to have two ounces of gin in water.

March 16th. The purging continued. The œdema of the face was quite gone. The urine was much less albuminous. His debility had increased. The tongue was getting brown and dry. Some tenderness was complained of on pressure over the abdomen. He was ordered sesquicarbonate of ammonia, bicar. bonate of soda, with tincture of opium, with chalk mixture in addition, if necessary ; and to take half an ounce of brandy in water every four hours. The abdomen was ordered to be fomented, and twelve leeches to be applied if the tenderness continued.

March 17. The abdominal tenderness was much increased; the purging was diminished. The leeches had been applied without relief. The tongue was dry and brown; and there were sordes on the teeth. Pulse 120 , very feeble. There was no abnormal heat of skin. A blister was ordered to be applied to the abdomen, and he was directed to continue the brandy every two hours. Beef-tea had been taken freely from the commencement.

March 18. All the symptoms were much worse. He still complained of great pain and tenderness of the abdomen, which the blister had not relieved. He took the brandy and beef-tea without difficulty. The purging was much diminished. $\mathrm{He}$ was ordered one grain of opium immediately, to be repeated every four or six hours, if necessray.

The abdomen became tympanitic; and he gradually sank exhausted on the following morning, the brain remaining unaffected to the last.

Autopsy. The abaiominal cavity contained a considerable quantity of sero-purulent fluid ; the peritoneal covering of the intestines showed patches of inflammation, but not of a plastic character. This was more particularly evident on the surface of the larger bowels. The peritoneal reflexion over the bladder and walls of the pelvis was evenly coated with a thin layer of pus. The same secretion existed also upon the serous covering below the liver, as well as upon the abdominal wall generally of the right side. The intestines themselves, with the 
exception of the peritoneal coat, were healthy. The appen dices epiploicæ of the colon were unusually large. Both kidneys were granular, but the left was both larger and more generally diseased than the right. The chest and head were not examined.

REMarks. From the granular condition in which the kidneys were found in the above case, it is probable that the primary disease had been of longer duration than a fortnight, as stated by the patient and his friends. The exposure to which he was subjected in his journey to the hospital was amply sufficient to give rise to the two secondary diseases consequent on the albuminuria, viz., diarrhœa and peritonitis.

Diarrhœa is not a very common secondary disorder attendant upon granular disease of the kidney in this country. In Scotland, however, according to Dr. Christison, as well as from my own observation, diarrhœa is a very common secondary at tendant upon this disease, often proving, as in the present instance, a very exhausting, obstinate complaint, and under which the patient not unfrequently succumbs.

of the secondary inflammation attacking the serous membranes, the order in which they most generally obtain, are pleuritis, then peritonitis, and least commonly pericarditis. In the above case, from the impoverished condition of the blond, the inflammation was of a very low character, as evidenced by the absence of all fibrinous adhesions, as well as by the thin, semi-formed condition of the pus, which was found spread over the peritoneal lining of the different portions of the abdominal walls.

\section{Tramsactions of 掘ramedres.}

\section{MIDLAND BRANCH.}

TWO CASES OF GANGRENE AFTER INJURY, IN WHICH AMPUTATION WAS SUCCESSFULLY PERFORMED.

By A. H. Dolman, Esq., Derby.

[Read March 22nd, 1860.]

HaviNg seen within the last six months two cases of gangrene, one of the foot, the other of the hand, in which amputation was performed, I thought a slight account of the two cases would not be uninteresting to you. The importance and anxiety inseparable in these serious cases cannot be overstated, for there is inevitable loss of limb, and very frequently, sad to relate, loss of life.

Both these patients are of the same age (40); and both were in good health up to the time of their respective injuries; and, I am happy to say, that although each has lost a limb and undergone a great amount of suffering, they are quite well.

Case I. Thomas Maddox, aged 40, a greengrocer, residing in Derby, was admitted into the Derbyshire General Infirmary, under the care of Mr. Gisborne, September 21, 1859. He was walking by the side of his horse and cart, when the former suddenly took fright, and ran away. Maddox, in attempting to stop the horse, was knocked down, and one of the cartwheels passed over both thighs. The vehicle was loaded at the time. He was brought a distance of nine miles to the in. firmary ; and, on examination being made, he was found to be suffering from comminuted fracture, with great displacement, of the left femur at the junction of the lower and middle thirds. He had also received a severe laceration of the right thigh. There was considerable spasm, and strong contraction of the muscles of the fractured limb. When this had subsided, the fracture was reduced; and a long straight splint was applied in the usual manner. He went on very well till the 27 th (the third day), when he complained of pain about the calf of the leg; the bandages were cut open, and some cooling lotion was applied.

Sept. 28th. The toes were rather dark coloured, and the thigh was enormously swollen, tense, and of universal dark brown colour, more particularly at the inner aspect, over the course of the vessels. The splint was removed, and the whole limb enveloped in cotton-wool. The temperature of the foot was rather low; but that of the leg and thigh was high. There were no marks of constriction on the limb, and no signs of the bandage having affected the circulation. A grain of opium was given to relieve the pain, and repeated when necessary.
Sept. 29th. A linseed poultice was applied to the thigh. The opium was ordered to be repeated twice a day.

Sept. 30th. He slept pretty well. The foot, anterior to the instep, was very dark coloured. He was ordered to have a pint of porter daily. The opium was repeated, and an ounce of cinchona mixture, with ammonia, was ordered to be taken three times a day.

Oct. 10th. He was somewhat weaker than he was at the date of last report. The anterior portion of the foot was gangrenous. The line of demarcation was tolerably distinct, just as if Nature was going to perform Chopart's operation. He was ordered four ounces of wine daily; and an ounce of compound tincture of cinchona, and sixteen grains of disul. phate of quinine were added to each pint of his mixture.

Oct. 13th. He was directed to have brandy when required.

Oct. 14th. The leg was swollen very much; fluctuation was perceptible opposite the calf. A free incision was made, and an immense quantity of offensive sanious matter, mixed up with air, was let out.

Oct. 20th. The foot was almost off; the leg was discharging enormously; the thigh was somewhat reduced in size, though still in an unhealthy state. It was evident that amputation of the limb was the only chance, but the condition of the thigh did not admit of it at present. There was considerable shortening, in consequence of the fracture being oblique, and as extension could not be applied.

Oct. 27th. The thigh being reduced in size, it was decided, on consultation, that the limb should be removed. Chloroform having been administered, a thorough examination was made it was found impracticable to operate below the knee. The limb was amputated just above the seat of fracture. A modification of Teale's operation was adopted; the difference being a long round anterior flap, instead of a rectangular one, the posterior short flap being cut accordingly. The stump was sewn up with iron sutures. His diet was not interfered with.

Nov. 1st. On dressing the stump, a large quantity of thin offensive matter was evacuated.

Nov. 3rd. I removed the sutures; and, as the stump was large and rather flabby, placed a bandage round it.

Nov. 11th. The stump had been dressed every other day since the last date. This morning, the ligature on the femoral artery came away.

Nov. 25th. He was still going on well; the stump was nearly healed. He was allowed to get up.

Dec. 1st. A small abscess formed on the posterior flap; it was opened with a bistoury, and a poultice was applied.

Dec. 19th. He was discharged cured.

On examination of the amputated limb, no lesion of the vessels or nerves could be detected. The coats of the posterior tibial artery (traceable to the internal malleolus) were thickened, and there was a good deal of deposit in the sheath. The anterior muscles of the leg had sloughed away, leaving the bones quite bare.

CASE Ir. Henry Whiting, aged 41, a labourer, residing at Doveridge, was admitted into the Derbyshire General Infirmary, January 3rd, 1860. Four days before admission, his right hand was drawn between the cogs of a thrashing machine, which inflicted, according to his statement, severe lacerations both on the dorsal and palmar aspects of the carpus. Medical assistance was soon procured, and the wound was stitched up. On admission into the Infirmary, the whole hand was found to be gangrenous, though there was no distinct line of demarcation. The arm was swollen and erythematous, with streaks of a dark colour extending up to the shoulder. Chloride lotion was applied to the hand, and oil to the arm; the whole was then enveloped in cotton-wool. He was ordered to have a pint of porter daily, and good diet. A draught of cinchona mixture with ammonia was given three times a day; and a grain of opium every night.

Jan. 4th. A great deal of offensive discharge from the hand took place. A large linseed poultice with charcoal was ordered to be applied, and frequently changed.

Jan. 11th. The line of demarcation was distinct; the whole arm was still inflamed, and tender to the touch.

Jan. 17th. As the arm was nearly reduced to its normal size, and there were healthy granulations at the line of demarcation, it was decided that amputation should be performed. Chloroform being administered, the limb was removed at the middle third of the forearm, by the flap operation in the usual way. Four arteries were tied. The stump was left open for a few hours; when it had become glazed, three wire sutures and a few narrow strips of plaster were applied. No change was made in the diet. 